\title{
Front Matter: Volume 9781
}

, "Front Matter: Volume 9781," Proc. SPIE 9781, Design-Process-Technology Co-optimization for Manufacturability X, 978101 (28 June 2016); doi: $10.1117 / 12.2239765$

SPIE. Event: SPIE Advanced Lithography, 2016, San Jose, California, United States 


\title{
Design-Process-Technology Co-optimization for Manufacturability $X$
}

\author{
Luigi Capodieci \\ Jason P. Cain \\ Editors
}

24-25 February 2016

San Jose, California, United States

Sponsored by

SPIE

Cosponsored by

Hitachi High Technologies America, Inc. (United States)

Published by

SPIE

Volume 9781 
The papers in this volume were part of the technical conference cited on the cover and title page. Papers were selected and subject to review by the editors and conference program committee. Some conference presentations may not be available for publication. Additional papers and presentation recordings may be available online in the SPIE Digital Library at SPIEDigitallibrary.org.

The papers reflect the work and thoughts of the authors and are published herein as submitted. The publisher is not responsible for the validity of the information or for any outcomes resulting from reliance thereon.

Please use the following format to cite material from these proceedings:

Author(s), "Title of Paper," in Design-Process-Technology Co-optimization for Manufacturability $X$, edited by Luigi Capodieci, Jason P. Cain, Proceedings of SPIE Vol. 9781 (SPIE, Bellingham, WA, 2016) Six-digit Article CID Number.

ISSN: 0277-786X

ISSN: 1996-756X (electronic)

ISBN: 9781510600164

Published by

SPIE

P.O. Box 10, Bellingham, Washington 98227-0010 USA

Telephone +1 3606763290 (Pacific Time) · Fax +1 3606471445

SPIE.org

Copyright @ 2016 , Society of Photo-Optical Instrumentation Engineers.

Copying of material in this book for internal or personal use, or for the internal or personal use of specific clients, beyond the fair use provisions granted by the U.S. Copyright Law is authorized by SPIE subject to payment of copying fees. The Transactional Reporting Service base fee for this volume is $\$ 18.00$ per article (or portion thereof), which should be paid directly to the Copyright Clearance Center (CCC), 222 Rosewood Drive, Danvers, MA 01923. Payment may also be made electronically through CCC Online at copyright.com. Other copying for republication, resale, advertising or promotion, or any form of systematic or multiple reproduction of any material in this book is prohibited except with permission in writing from the publisher. The CCC fee code is 0277-786X/16/\$18.00.

Printed in the United States of America.

Publication of record for individual papers is online in the SPIE Digital Library.

\section{SPIE. DIGITAL}

Paper Numbering: Proceedings of SPIE follow an e-First publication model. A unique citation identifier (CID) number is assigned to each article at the time of publication. Utilization of CIDs allows articles to be fully citable as soon as they are published online, and connects the same identifier to all online and print versions of the publication. SPIE uses a six-digit CID article numbering system structured as follows:

- The first four digits correspond to the SPIE volume number.

- The last two digits indicate publication order within the volume using a Base 36 numbering system employing both numerals and letters. These two-number sets start with 00, 01, 02, 03, 04, $05,06,07,08,09,0 A, 0 B \ldots$ OZ, followed by 10-1Z, 20-2Z, etc. The CID Number appears on each page of the manuscript. 


\title{
Contents
}

\author{
vii Authors \\ xi Conference Committee
}

\section{SESSION $1 \quad$ LAYOUT OPTIMIZATION AND DESIGN RESTRICTIONS}

978102 Toward the $5 \mathrm{~nm}$ technology: layout optimization and performance benchmark for logic/SRAMs using lateral and vertical GAA FETs (Invited Paper) [9781-1]

978103 Structural design, layout analysis and routing strategy for constructing IC standard cells using emerging 3D vertical MOSFETs [9781-2]

978104 Directed self-assembly aware restricted design rule and its impact on design ability [9781-3]

978105 Integrated routing and fill for self-aligned double patterning (SADP) using grid-based design [9781-4]

978106 Integrated layout based Monte-Carlo simulation for design arc optimization [9781-5]

978107 Impact of EUV patterning scenario on different design styles and their ground rules for $7 \mathrm{~nm} / 5 \mathrm{~nm}$ node BEOL layers [9781-6]

\section{SESSION 2 LAYOUT ANALYTICS}

978108 Methodology for analyzing and quantifying design style changes and complexity using topological patterns (Invited Paper) [9781-7]

978109 Methodology to extract, data mine and score geometric constructs from physical design layouts for analysis and applications in semiconductor manufacturing [9781-8]

9781 OA Using pattern enumeration to accelerate process development and ramp yield [9781-9]

9781 OB Optimization of self-aligned double patterning (SADP)-compliant layout designs using pattern matching for 10nm technology nodes and beyond [9781-10]

\section{SESSION 3 DESIGN AND LITHO OPTIMIZATION: JOINT SESSION WITH CONFERENCES 9780 AND 9781}

9781 OC Hybrid hotspot detection using regression model and lithography simulation [9781-12] 
9781 OD Variability-aware compact modeling and statistical circuit validation on SRAM test array [9781-13]

$9781 \mathrm{OE} \quad$ Impacts of process variability of alternating-material self-aligned multiple patterning on SRAM circuit performance [9781-14]

9781 OF Modeling interconnect corners under double patterning misalignment [9781-15]

9781 OG Model-based CMP aware RC extraction of interconnects in 16nm designs [9781-16]

\section{SESSION 5 HOTSPOT DETECTION AND REMOVAL}

$9781 \mathrm{OH} \quad$ Automatic layout feature extraction for lithography hotspot detection based on deep neural network (Invited Paper) [9781-17]

97810 Pattern-based DTCO flow for early estimation of lithographic difficulty using optical image processing [9781-18]

9781 0J A random approach of test macro generation for early detection of hotspots [9781-19]

9781 OK Hotspot detection and removal flow using multi-level silicon-calibrated CMP models [9781-20]

9781 OL Migrating from older to newer technology nodes and discovering the process weak-points [9781-21]

\section{SESSION 6 MULTIPLE PATTERNING AND DIRECTED SELF-ASSEMBLY}

$97810 \mathrm{M}$ Triple/quadruple patterning layout decomposition via novel linear programming and iterative rounding [9781-22]

9781 ON Design strategy for integrating DSA via patterning in sub-7 $\mathbf{~ m}$ interconnects [9781-23]

$978100 \quad$ Enablement of DSA for VIA layer with a metal SIT process flow [9781-24]

9781 OP Layout decomposition and synthesis for a modular technology to solve the edgeplacement challenges by combining selective etching, direct stitching, and alternatingmaterial self-aligned multiple patterning processes [9781-25]

$97810 Q \quad$ Metal stack optimization for low-power and high-density for N7-N5 [9781-45]

9781 OR The new analysis method of PWQ in the DRAM pattern [9781-27] 
9781 OS Verification and application of multi-source focus quantification [9781-28]

9781 OT A comparative study on the yield performance of via landing and direct stitching processes for 2D pattern connection [9781-29]

9781 OU Estimate design sensitivity to process variation for the 14nm node [9781-30]

INTERACTIVE POSTER SESSION

9781 OV Interlayer design verification methodology using contour image [9781-26]

9781 OW Design space exploration for early identification of yield limiting patterns [9781-31]

9781 OX Design technology co-optimization for 14/10nm metal1 double patterning layer [9781-32]

9781 OY An integrated design-to-manufacturing flow for SADP [9781-33]

$97810 Z$ Using pattern analysis methods to do fast detection of manufacturing pattern failures [9781-34]

978110 Electron-beam lithography with character projection exposure for throughput enhancement with line-edge quality optimization [9781-35]

978111 Rapid recipe formulation for plasma etching of new materials [9781-36]

978112 Expanding the printable design space for lithography processes utilizing a cut mask [9781-37]

978113 Characterization of shallow trench isolation CMP process and its application [9781-38]

978114 Advanced DFM application for automated bit-line pattern dummy [9781-39]

978115 Wafer hotspot prevention using etch aware OPC correction [9781-40]

978116 Building block style recipes for productivity improvement in OPC, RET and ILT flows [9781-41]

978117 Hybrid pattern matching based SRAF placement [9781-42]

978118 LELE CD bias offset monitor through OVL measurement [9781-43] 
Proc. of SPIE Vol. $9781978101-6$

Downloaded From: https://www.spiedigitallibrary.org/conference-proceedings-of-spie on 25 Apr 2023 Terms of Use: https://www.spiedigitallibrary.org/terms-of-use 


\title{
Authors
}

Numbers in the index correspond to the last two digits of the six-digit citation identifier (CID) article numbering system used in Proceedings of SPIE. The first four digits reflect the volume number. Base 36 numbering is employed for the last two digits and indicates the order of articles within the volume. Numbers start with 00, 01, 02, 03, 04, 05, 06, 07, 08, 09, OA, OB...0Z, followed by 10-1Z, 20-2Z, etc.

\author{
Ali, Hussein, OW \\ Al-Imam, Mohamed, 0J \\ Amir, Nuriel, 18 \\ Asada, Kunihiro, 10 \\ Asthana, Abhishek, 12 \\ Baert, R., OQ \\ Bahr, Mohamed, 14 \\ Baldick, Ross, OM \\ Ban, Yongchan, OG \\ Batarseh, Fadi, 09 \\ Bekaert, Joost, ON \\ Bonnecaze, Roger T., 11 \\ Bravo, Jaime, 09, OK \\ Cain, Jason P., 08, 01 \\ Capodieci, Luigi, OB \\ Cecil, Tom, 16 \\ Chang, Jinman, OR \\ Chee, Jansen, OK \\ Chen, Alek C., 07 \\ Chen, Ao, 15 \\ Chen, Howard, 18 \\ Chen, Kuang-Han, 0G, OK, 13 \\ Chen, Xiaohui, 16 \\ Chen, Yijian, 03, OE, OP, OT \\ Chen, Ying, $0 X$ \\ Chen, Yulu, 04 \\ Cheng, Qi, OE \\ Chiou, Tsann-Bim, 07 \\ Chiv, Yang-Chih, 13 \\ Choi, Bumjin, OR \\ Choi, Eunjoo, OG \\ Choi, Jinwoo, OV \\ Choi, Seiryung, OV \\ Chopra, Meghali, 11 \\ Clevenger, Larry, 06 \\ Croes, Kris, ON \\ Culp, James, 06 \\ Curtice, Mark, 12 \\ Debacker, P., $O Q$ \\ Deeth, Steven, 16 \\ Dehaene, Wim, ON \\ Ding, Hua, OA, OL, 0Z, 13 \\ Du, Chunshan, OW \\ Duan, Yingli, OX \\ Dusa, Mircea, 07, 0Q \\ Ekerdt, John, 11 \\ ElManhawy, Wael, Ol, OJ, OW \\ Fakhry, Moutaz, Ol \\ Farys, Vincent, 0O, OU
}

Feng, Jui-Hsuan, 12

Fenouillet-Beranger, C., 00

Firouzi, F., OQ

Gao, Shao Wen, 12

Gbondo-Tugbawa, Tamba, 0G, OK, 13

Gemmink, J.-W., OS

Gennari, Frank E., 08, 09

Gerousis, V., OQ

Gower-Hall, Aaron, 0K, 13

Gronheid, Roel, ON

Hamed Fatehy, Ahmed, OY

Hamouda, Ayman, 15

Han, Daehan, OR

Han, Sang Min, OG, OK

Han, Ting, 03, OE, OP

Harb, Mohammed, OJ

Hasan, T., OS

Hong, Aeran, OR

Hong, Chuyang, 03, OE

Hong, Hyeongsun, OR, OV

Hong, Sid, OW

Hsing, Henry, 18

Hsu, Danny, OW

Hui, Colin, OK

Hurat, Philippe, 09

Huynh-Bao, Trong, 02

Hwang, Sungwook, OJ

Hyun, Daijoon, OF

Ikeda, Makoto, 10

Ikeno, Rimon, 10

Jin, Gyoyoung, OR

Jourlin, Y., OS

Kabeel, Aliaa, OW

Kang, Jinyoung, OR

Kang, Minsoo, OJ

Kang, Namjung, OV

Karageorgos, Evangelos, ON

Karageorgos, loannis, ON

Katakamsetty, Ushasree, OK

Kim, Cheolkyun, 14

Kim, Chin, OJ

Kim, Hyoung Jun, OR

Kim, Hyoungsun, OR

Kim, Ryoung-han, 04

Kim, Seoksan, OV

Kim, Taeheon, OR

Kim, Yonghyeon, OR

Kimura, Taiki, OC

Kotani, Toshiya, $\mathrm{OH}$ 
Kotb, Rehab, OY

Krishnamoorthy, Karthik, 09

Kwa, Denny, 16

Kwan, Joe, Ol, 0J, OW

Lai, Ya-Chieh, 08, 09, 0A, OL, OZ

Landié, Guillaume, OU

Lee, Brian, OK, 13

Lee, Jeemyung, 05

Lee, Jong-hyun, OJ

Lee, Joosung, OR

Lee, Kweonjae, OR

Lee, Kyupil, OV

Lee, Kyusun, OR

Lee, Robben, OW

Lee, Seongmin, 05

Lee, Sho Shen, 18

Le-Gratiet, B., OS

Lei, Junjiang, $\mathrm{OX}$

Li, Flora, 13

Li, Helen, OW, 13

Li, Yongfu, OK

Liebmann, Lars, 06

Lin, Chia Ching, 18

Lin, Yibo, OM

Lio, En Chuan, 18

Liv, Hongyi, 03, OP

Liu, JinBing, 13

Liu, Kince, 18

Liv, Qing Wei, OL

Liu, ZhengFang (Square), OW, 13

Lucas, Kevin, 16

Lutich, Andrey, 17

Madhavan, Sriram, 09, OB

Madkour, Kareem, OI, OJ, OW

Maruyama, Satoshi, 10

Matsunawa, Tetsuaki, $\mathrm{OC}, \mathrm{OH}$

Matti, L., OQ

Mclntyre, G., OQ

Meng, Xiaodong, 16

Mercha, Abdelkarim, 02

Mita, Yoshio, 10

Nojima, Shigeki, OC, $\mathrm{OH}$

Omran, Ahmed, 17

Pack, Bob, 09

Pan, David Z., OC, OM

Pang, Jenny, OA, OL

Park, Jaekyun, OV

Park, Sungmin, OV

Pathak, Piyush, 09

Power, Dave, 15

Prentice, C., OS

Qiao, Ying, OD

Raghavan, P., OQ

Ronse, K., OQ

Ryckaert, Julien, 02, ON, OQ

Sakhare, Sushil, 02

Salama, Mohamed, 12, 15

Schneider, L., 00

Schroeder, Uwe Paul, 09, 0B, 17

Serret, E., 00
Shao, Dongbing, 06

Shao, Feng, OX

Sherazi, S. M. Y., OQ

Shi, Xue Long, OL

Shim, Minyoung, OV

Shim, Myoungseob, OV

Shin, Jaepil, OV

Shin, Tae Hyun, 14

Shin, Youngsoo, 05, OF

Simiz, J.-G., OS

Somani, Shikha, 09

Song, Youngsoo, 05

Spanos, Costas J., OD

St. John, Matt, 16

Staals, F., OS

Stucchi, Michele, ON

Su, Xiaojing, OX

Su, Yajuan, OX

Sun, Mason, $\mathrm{OZ}$

Sung, Hyunju, OV

Sweis, Jason, 08, 09, 0A, 0L, OZ

Tel, W. T., OS

Thean, Aaron, 02

Tishchenko, A., OS

Tokei, Z., OQ

Trivkovic, D., OQ

Tsai, Mengfeng, OA, OL

Tseng, Shih-En, 07

Tung, Maryann C., ON

Vandenberghe, Geert, ON

Verkest, Diederik, 02, OQ

Wambacq, Piet, 02

Wan, Jinyin, 16

Wandell, Jerome, 12

Wang, Amy, OA

Wang, Chang Mao, 18

Wang, Jeff, $\mathrm{OZ}$

Wang, Jessie, $0 Z$

Wang, JinYan, OW

Wang, Lynn T.-N., OB

Wang, Tom, 16

Wang, Wei-Long, 09

Wei, Yayi, OX

Wilkinson, William, 12

Wong, H.-S. Philip, ON

Wong, Robert, 06

Woo, Youngtag, OB

Word, James, OY

Wu, Linghui, 16

$X U$, Jessy, OA, OL

$\mathrm{XU}$, Xiaoqing, $\mathrm{OM}$

Yang, Ellyn, OL

Yang, Hyunjo, 14

Yang, Jae-seok, OJ

Yu, Bei, OM

Zeng, Jia, OB

Zhang, ChunLei, 13

Zhang, Recco, OW, OX

Zhang, Yifan, OA, OL, OZ

Zhang, Zizhuo, 11 
Zhao, Evan, $\mathrm{OZ}$

Zhou, Jun, 03, OP, OT

Zhuang, Lei, 06

Zhuang, Linda, OA, OL

Zou, Elain, OW

Proc. of SPIE Vol. $9781978101-9$

Downloaded From: https://www.spiedigitallibrary.org/conference-proceedings-of-spie on 25 Apr 2023 Terms of Use: https://www.spiedigitallibrary.org/terms-of-use 
Proc. of SPIE Vol. $9781978101-10$

Downloaded From: https://www.spiedigitallibrary.org/conference-proceedings-of-spie on 25 Apr 2023 Terms of Use: https://www.spiedigitallibrary.org/terms-of-use 


\section{Conference Committee}

Symposium Chair

Mircea V. Dusa, ASML US, Inc. (United States)

Symposium Co-Chair

Bruce W. Smith, Rochester Institute of Technology (United States)

Conference Chair

Luigi Capodieci, KnotPrime Inc. (United States)

Conference Co-Chair

Jason P. Cain, Advanced Micro Devices, Inc. (United States)

Conference Program Committee

Robert Aitken, ARM Inc. (United States)

Fang-Cheng Chang, Cadence Design Systems, Inc. (United States)

Neal V. Lafferty, Mentor Graphics Corporation (United States)

Lars W. Liebmann, IBM Corporation (United States)

Ru-Gun Liu, Taiwan Semiconductor Manufacturing Co. Ltd. (Taiwan)

Mark E. Mason, Texas Instruments Inc. (United States)

Andrew R. Neureuther, University of California, Berkeley (United States)

Shigeki Nojima, Toshiba Corporation (Japan)

David Z. Pan, The University of Texas at Austin (United States)

Chul-Hong Park, SAMSUNG Electronics Co., Ltd. (Korea, Republic of)

Michael L. Rieger, Synopsys, Inc. (United States)

Vivek K. Singh, Intel Corporation (United States)

Chi-Min Yuan, Freescale Semiconductor, Inc. (United States)

John L. Sturtevant, Mentor Graphics Corporation (United States)

Session Chairs

1 Layout Optimization and Design Restrictions

Luigi Capodieci, KnotPrime Inc. (United States)

Jason P. Cain, Advanced Micro Devices, Inc. (United States)

2 Layout Analytics

David Z. Pan, The University of Texas at Austin (United States)

Luigi Capodieci, KnotPrime Inc. (United States) 
3 Design and Litho Optimization: Joint Session with Conferences 9780 and 9781

Daniel Sarlette, Infineon Technologies Dresden (Germany)

4 Circuit Modeling

Chi-Min Yuan, Freescale Semiconductor, Inc. (United States)

Robert Aitken, ARM Inc. (United States)

5 Hotspot Detection and Removal

Neal V. Lafferty, Mentor Graphics Corporation (United States)

Chul-Hong Park, SAMSUNG Electronics Co., Ltd. (Korea, Republic of)

6 Multiple Patterning and Directed Self-Assembly

Michael L. Rieger, Synopsys, Inc. (United States)

Vivek K. Singh, Intel Corporation (United States)

$7 \quad$ Design Interaction with Metrology: Joint Session with Conferences

9778 and 9781

Alexander Starikov, I\&I Consulting (United States)

Jason P. Cain, Advanced Micro Devices, Inc. (United States)

8 Process and Yield Modeling

Shigeki Nojima, Toshiba Corporation (Japan)

Fang-Cheng Chang, Cadence Design Systems, Inc. (United States) 